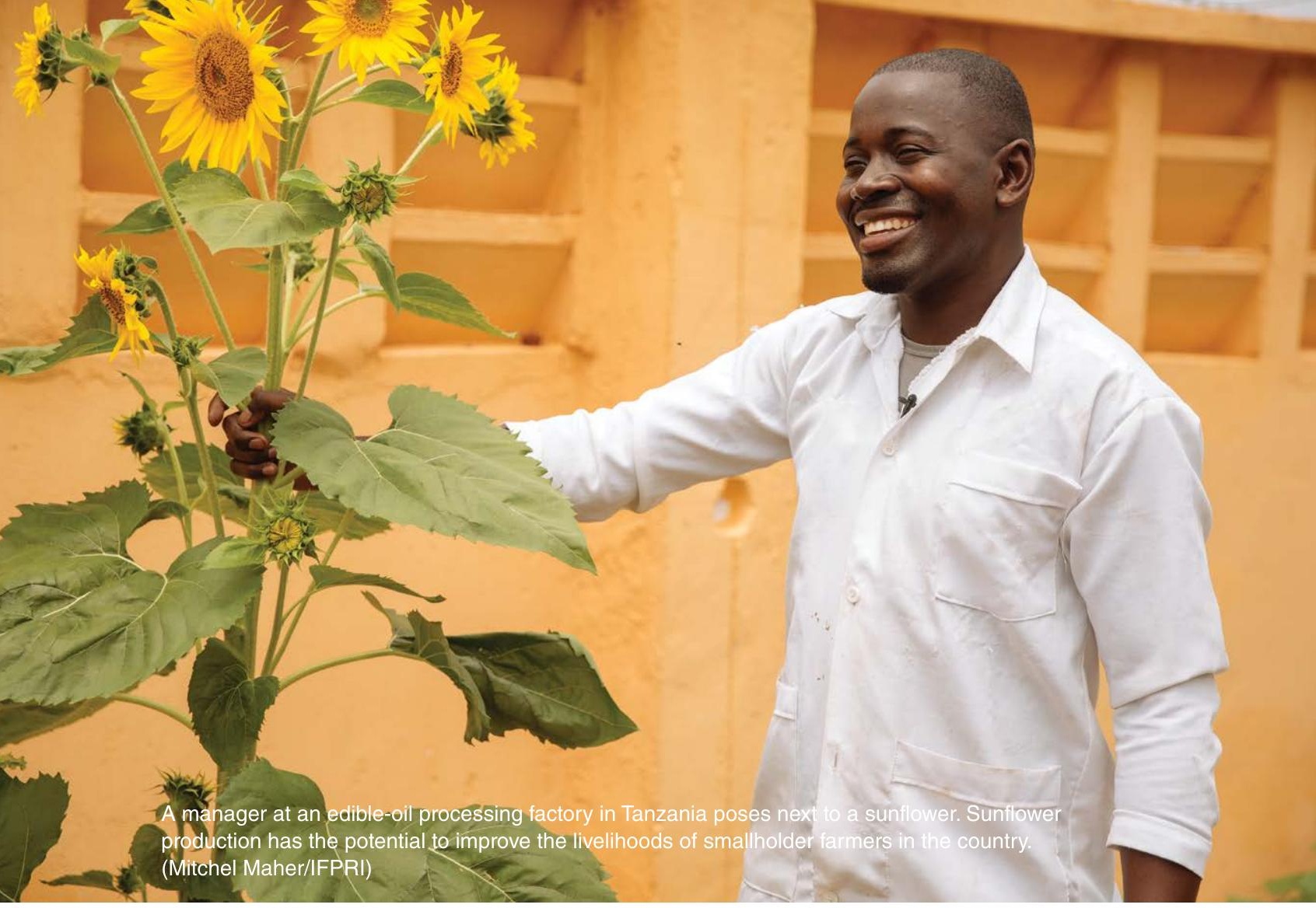

20

\title{
The Way Forward for Nutrition-driven Agriculture
}

Shenggen Fan, Sivan Yosef* and Rajul Pandya-Lorch International Food Policy Research Institute (IFPRI), Washington, DC, USA

* Corresponding author: s.yosef@cgiar.org 
This volume has highlighted the important links between agriculture and nutrition, both direct and indirect, both theoretical and practical. It has explored these relationships through various frameworks, such as value chains, programs and policies, as well as through diverse perspectives, such as gender. It has assessed the impacts of various agricultural interventions and policies on nutrition, including those that integrate behavior change communication or social protection, and profiled the up-and-down journeys of countries such as Bangladesh, China, Ethiopia, India, and Malawi in integrating nutrition into agriculture policies and program interventions. It has highlighted successes such as biofortification, the integration of behavior change communication and gender equality into existing agricultural interventions, and agriculture's role in improving household access to nutritious foods and diet diversity. It has analyzed challenges such as climate and environmental change, undernutrition, and obesity. And it has pondered big questions, such as how to build capacity, engage with the private sector, participate in the big data revolution, and foster strong governance and leadership throughout agriculture and nutrition.

The volume has conveyed that we know far more about the ways in which agriculture and nutrition interact than we did just 5-10 years ago, when interest in the agriculture-nutrition nexus began to really take off. More and more nutrition-driven agricultural programs are well designed, and the evaluations of their impact are more rigorous, relying on superior sample sizes and data analysis approaches, as well as clearer program impact pathways. Policy makers are also paying more attention, and devoting more policy space, to agriculture and nutrition. Whether this rhetoric translates into action remains to be seen in many low-income countries.

Despite the wide range of issues and perspectives appearing in this book, several strong themes emerge that provide interesting insights on how to move agriculture-nutrition forward in the coming years.

\section{Start with Clear Nutrition Goals}

It may seem obvious that in order to achieve truly nutrition-driven agricultural policies, programs, investments, and strategies, we need to explicitly integrate nutrition into their design. Yet many 'nutrition-sensitive' agricultural interventions or policies do not use clear, measurable nutrition goals as indicators of success. 'Nutrition driven' sends the clear message that nutrition outcomes are being proactively included as explicit goals of agricultural programs and interventions. These outcomes include changes in anthropometry, improved micronutrient status, increased dietary diversity, and/or increased consumption of nutritious foods. Relevant, nutritionfriendly goals also include achieving gender parity in decision-making power over agricultural resources and household income. In this latter case, the impact pathways to nutrition - and to reaching, benefiting, or empowering women need to be clear and quantifiable (with this reasoning applying to other types of interventions as well, such as social protection, as described by Gilligan in Chapter 10). Simply having women participating in a program does not necessarily lead to nutrition outcomes, and even when women do benefit, they are not necessarily empowered (Chapter 6). Within nutrition-driven programs and policies, nutrition is not merely an afterthought, but rather, a primary, strategic goal.

\section{Move Beyond Staples}

Many agricultural policies in high- and lowincome countries were originally designed to address undernourishment, in terms of energy intake, and food security in emergency settings. As a result, national policies tend to favor the production of staple foods; for example, wheat and rice in India (Chapter 17) and maize in Malawi (Chapter 18). In countries such as Bangladesh, the price fluctuations for non-rice crops are much larger than for rice, indicating a high level of market-induced risks for farmers attempting to move out of staples (Chapter 15). Agricultural policies also make nutrient-dense foods such as animal-source products, fruits, and vegetables cost-prohibitive for poor households to both produce and consume. In fact, the high expense of nutritious foods for the poor provides perhaps the best reason for nutritionfocused agricultural development since 'other economic sectors may well drive income growth, but only food policies can influence the 
affordability of nutritious foods' (Chapter 2). Reducing or eliminating agricultural distortion policies can be a step forward in lowering the costs of nutritious foods. But agricultural subsidies often also have a social protection aim to them, since farmers make up a large share of the poor population (Chapter 8 ). Thus, any policy shift to eliminate distortions should be accompanied by equal measures to ensure that poor agricultural households are not left behind. One option is to shift the focus of agricultural subsidies to support the production, processing, transportation, and marketing of nutritious foods. This support can come in the form of increased research and development on nutrient-dense crops, higher investment in infrastructure underlying the value chains of non-staple foods, and income support to poor and vulnerable farming households growing these crops.

\section{Include the Minimum Package in Any Intervention}

Nutrition-sensitive agricultural interventions are especially effective when they include behaviorchange communication and can even improve child nutrition outcomes such as dietary diversity, nutrient intakes, anemia, diarrhea, and wasting when they integrate health-seeking and water, sanitation, and hygiene practices, or provide fortified products in areas where it is difficult to access nutrient-rich foods (Chapter 9). Thus, agricultural programs seeking to ensure an impact on nutrition should consider, at the very minimum, integrating three components: (i) strong behavior-change communication; (ii) activities that empower women but also promote gender equality within households and communities; and (iii) the provision of micronutrient-fortified products targeted to children and pregnant women. In Senegal, for example, a dairy value chain project distributed micronutrient-fortified yogurt as an incentive to female dairy farmers for increasing their milk production. The strategy was coupled with behavior change communication on infant and young child feeding. An evaluation found that children of participating farmers who received both behavior change communication and the fortified yogurt had greater increases in hemoglobin than the control group that had received just behavior change communication (Chapter 6).

\section{Be Prepared for Unintended Consequences}

Nutrition and agriculture interventions and policies are implemented in the real world. Oftentimes, evaluations reveal unintended impacts after the fact. For example, implementing agricultural interventions to boost household income may unintentionally lead households to use that income to purchase commercially processed foods, raising the risk of malnutrition due to the consumption of obesogenic foods (Chapter 7). Including women in agricultural interventions can impact their time for child feeding and care (Chapters 6 and 9). Targeting a safetynet program to poor households may be effective in reducing poverty, but not in boosting agricultural growth (Chapter 10). In China, large-scale nutrition programs have focused on infants and children, leading to the unintended consequence of seniors and the urban poor being left behind (Chapter 19). Up to $30 \%$ of people aged 60 or older in China are malnourished (Peking University China Health and Retirement Longitudinal Study, 2017). In India, price and marketing support to staple-grain production has reduced farmers' incentives to grow nutrient-rich coarse grains and pulses, reducing the availability of these crops (Chapter 17).

It is for this reason that Raiten and Combs (Chapter 7) advocate for a systems approach to nutrition, through nutrition ecology, which can help program designers assess all of the different local factors that can affect targeted communities, including differences in household members' access to nutrition and health, women's time availability, land use and availability, crop responses, and more.

\section{Capitalize on, Not Shy Away from, Large-scale Changes}

The world is changing rapidly. Many countries are facing significant demographic shifts such as urbanization, rapid market transformation, and, as a result, rises in levels of obesity and other 
non-communicable diseases. Climate and environmental change threatens the stability and productivity of agricultural systems. It is up to the international development community and more so, to national policymakers, to capitalize on these changes, which will occur anyway, to reinvent food and agricultural systems to deliver nutrition outcomes. Actions in this regard can include leveraging longer rural-urban and peri-urban-urban value chains to promote the retention and increase of nutrition (Chapter 3) or tasking crop breeders to combine nutritional traits with environmental traits, such as tolerance to drought and salinity, as well as to seasonal availability, so that multiple benefits can be gained (Chapter 4).

\section{Build Up Incentives}

What is the incentive for an agricultural extension agent in Brazil to incorporate nutrition messaging into her daily workplan? What will prompt a mother of four living on $\$ 1$ a day in Thailand to shift part of her household food budget to pulses instead of rice? What will entice a small private-sector seed company in Bangladesh to start offering zinc-biofortified rice seeds to farmers? (The answer to this real-life conundrum has been for HarvestPlus to both guarantee a market for a portion of the private-sector production and subsidize the price for any seed that the private-sector markets directly to farmers; see Chapter 5 for more).

The various actors in the agricultural system, and the institutions within which they carry out their work, need incentives to work across sectors and outside their comfort zones. The private sector needs to be incentivized to promote public nutrition goals - in Chapter 11, Haddad offers numerous examples of policy carrots and policy sticks that governments can deploy to align these incentives. Undoubtedly, the strongest incentive for the private sector is market demand for its products. Building consumer demand for nutritious foods can impact actors all along the value chain to shift their efforts toward promoting nutrition. As Allen et al. point out in Chapter 3, consumer demand can be built up by educating the public on the value of nutrition and health through information campaigns, or by adding 'visible value' to products such as labeling their nutrient composition or promoting their high quality standards to distinguish them from their competitors. When these efforts fall short, then public policy can play a role. For example, distortions against producing and consuming nutritious foods can be removed. Governments can also mount their own nutrition education campaigns, support research and development of nutritious foods, and invest in the development of the various components that make nutrition-driven value chains possible, such as rural roads, electricity, nutrition extension, and cold chains.

Governments themselves also need incentives. In Malawi, for example, the success of the Ministry of Agriculture, Irrigation and Water is measured by maize yields, not nutrition outcomes; thus, it is important to consider how agriculture ministries can be persuaded to improve diets and malnutrition (Chapter 18). Gillespie and Nisbett (Chapter 12) find that there is strong support among professionals working in nonhealth sectors for mandatory mechanisms for collaboration; many of these professionals want to promote nutrition but are lacking management support systems and incentives to do so.

\section{Reconsider Old Assumptions}

Breaking down and rebuilding an entire food and agricultural system may require shattering some old assumptions too. For example, does the singular focus on women within agriculturenutrition interventions work? Malapit (Chapter 6) suggests that focusing on the relationship between women and men is more optimal. This approach is already being taken up in Bangladesh, where the government-led ANGeL project is training both male and female beneficiaries so that they can make joint decisions on agricultural production and marketing within their households (Chapter 15).

In another example, many proponents of multisectorality advocate for integrating nutrition interventions into agricultural programs. But does this approach run the risk of making cross-sectoral programs too burdensome and complex? Is true integration really necessary, or can co-locating agriculture and nutrition 
interventions, i.e. carrying them out within the same community, be just as effective (Chapter 9)? The answers to these crucial questions require more dedicated research.

Finally, are multinational companies, with whom there are high levels of distrust among the nutrition community, the only viable players in public-private partnerships on nutrition? Small- and medium-sized businesses in horticulture and aquaculture, for instance, can play a huge role in the global production and dissemination of affordable and profitable nutritious foods. Furthermore, there is a wide array of resources that can help reduce the risk of embarking on a nutrition-focused partnership with the private sector, including accountability measures, conflict of interest guidelines, and more (Chapter 11).

\section{Don't Forget an Enabling Environment}

In food and agricultural systems, the law can be a powerful tool for setting the 'rules of the game'. In China, for example, legal mechanisms to promote public health and nutrition have faced challenges since the late 1980s and are only now returning to the forefront of nutrition strategy (Chapter 19). The law can permeate all areas of nutrition. For instance, in order for biofortification to be truly scaled, it needs to be recognized among global normative and regulatory agencies (Chapter 5). Setting quality standards for locally produced food products, such as fortified baby cereals, can go a long way in building up trust in nutritious foods among local populations and reducing the price of a healthy diet (Chapter 2).

An enabling environment can have farreaching effects. Policies on how to manage big data, including ensuring privacy, security, and clear ownership and regulating new technologies can yield standards that are jointly understood by different sectors and disciplines (Chapter 14). An enabling environment can also make capacity-building efforts possible, including providing channels for formal education focusing on agriculture-nutrition links, nutrition training for agricultural extension agents, and multi-sectoral research opportunities (Chapter 13).
Implementers of Ethiopia's Agricultural Growth Program, for instance, have benefited from an enabling environment that has allowed them to focus the first year of their work on creating capacities to implement a nutrition-sensitive agricultural strategy (Chapter 16).

An enabling environment can also help develop markets, especially key when considering that market access is a consistent and large modifying effect of the impacts of agriculture on nutrition outcomes, particularly dietary diversity (Chapter 9). The supporting environment for markets can include building infrastructure, such as roads and energy (Chapter 3 ). The price of inaction in this area is great. For example, the lack of a supportive environment for market connectivity and cold chains in India has limited the agricultural sector's diversification to higher value products that can improve diets and incomes (Chapter 17).

But an enabling environment also goes beyond policies. It includes the effective communication of knowledge and evidence, political commitment, leadership, capacity, financing, and governance (Chapter 12). Strong governance is particularly important in the case of power imbalances, such as between governments and multinational companies. Leadership is also crucial. While individual nutrition champions are certainly needed, sustainable leadership may exist in networks of individuals that can be held accountable by those suffering most from malnutrition. Additionally, leadership throughout nutrition, agriculture, and food systems, including leaders working at the ground and executive levels, can collectively contribute to systemic leadership (Chapter 12).

\section{And Finally, Fill Some Knowledge Gaps - and Then Fill Some More}

Perhaps the most salient theme of this volume is the need for more evidence. High-quality research has already suggested what could possibly work for agriculture-nutrition. For example, integrating behavior change communication into existing agricultural interventions can make them more effective in impacting nutrition (Chapter 9). Research has also demonstrated the efficacy of biofortified crops such as iron bean 
and iron pearl millet in improving nutritional status (Chapter 5). And new findings suggest that agricultural growth may be more likely to reduce stunting in Africa than in Asia, because stunting in Africa is more closely associated with poverty, with farming, and with rural livelihoods (Chapter 2). Equally as important, there are indications that evidence is oftentimes utilized beyond the research community. For example, in Ethiopia, findings from the Demographic and Health Survey and various landmark publications such as The Lancet helped push policymakers to go beyond food production to address malnutrition (Chapter 16). Thus, it is time to collect far more knowledge and lessons.

More research is needed on the sustainability of agriculture-nutrition interventions: in the long term, do participants of integrated interventions maintain the practices they adopted or keep the assets they built up? More work is needed on how to scale up successes and at what cost. Insights are needed on the political economy of linking the agriculture and nutrition sectors together, including analyzing power dynamics among various stakeholders and interest groups. More research is needed on how to assess the success of interventions with outcomes that span disciplines (Chapter 9). Cost-benefit analyses of nutrition-sensitive agricultural policies are lacking. The evidence on the impact of food value chains also remains limited (Chapter 3). Region-specific evidence is also sorely needed. For example, studies that analyze the nutrition impacts of specific agricultural policies are mostly lacking for Africa and the Middle East (Chapter 8).

Closely tied to research is the need for highquality data (Chapter 14). Most low- and middleincome countries, for example, do not document or publish figures on their expenditures on specific agricultural investments (Chapter 8 ). At the global level, data on production and pricing of horticulture is particularly scarce, a key challenge when considering the importance of fruits and vegetables for healthy diets. These are just a few examples of the wide gaps in knowledge within the agriculture-nutrition nexus and a call to action to researchers to begin closing them.

\section{The Start of Something New}

The many unknowns within the agriculturenutrition nexus are exactly what make it the pre-eminent field to be in. This volume has highlighted the pockets of advances in research, policy, and programs around the world during the past 5-10 years. Looking ahead to the next 5-10 years is an equally exciting prospect. During the next decade, findings from recentlyimproved program evaluations are sure to reveal new insights on which nutrition indicators, agricultural interventions and policies can reasonably affect, for which household members, and how and why. Students graduating with theoretical and practical knowledge of the impacts of agriculture on nutrition will launch careers where they can conduct research, design policies, and implement programs that have far-reaching effects on nutritionally-vulnerable people. And communities themselves will find ways of holding leaders and various actors accountable for advances in nutrition. These positive developments can become a reality as long as we continue to invest in knowledge, people, and institutions going forward.

The agricultural and food system is at a crossroads. Pervasive malnutrition, compounded by new challenges such as climate change and urbanization, make the current system untenable. If diets have been radically transformed for the worse in just a decade or two, as seen in the global obesity epidemic, why can't they be transformed again for the better within another decade? Indeed, with continued commitment to the agriculture and nutrition nexus, we may soon finally break the cycle of malnutrition for this and future generations.

\section{Reference}

Peking University (2017) China Health and Retirement Longitudinal Study. Available at: http://charls.pku. edu.cn/en (accessed 16 April 2018). 